\title{
EDUCACIÓN Y BIBLIOTECA, UNA REVISTA PROFESIONAL PARTICULAR
}

Javier Pérez Iglesias

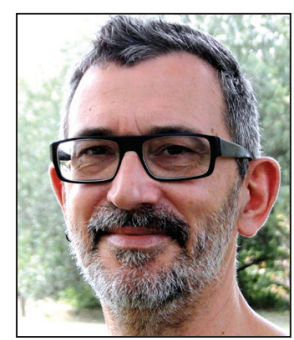

Javier Pérez-Iglesias es bibliotecario en la Universidad Complutense de Madrid. Trabaja en el equipo de Apoyo a la Docencia y a la Investigación y su actividad está relacionada con el open access, la gestión del patrimonio digital y alfin. Antes trabajó en la Universidad de Cantabria en el área de ciencia y tecnología de la Biblioteca. Durante tres años, de agosto de 2009 a julio de 2012, ha sido el responsable de la cooperación universitaria y científica en la Agencia Española de Cooperación Internacional para el Desarrollo (Aecid) del MAEC. Entre 1996 y 2001 fue director de la revista Educación y biblioteca.

http://orcid.org/0000-0003-1097-9366

Universidad Complutense de Madrid, Biblioteca Edif. Multiusos 1, Prof. Aranguren, s/n. 28040 Madrid, España jperezi@buc.ucm.es

\section{Resumen}

La revista Educación y biblioteca ha acompañado a las bibliotecas públicas españolas en el gran cambio que han llevado a cabo a lo largo de tres décadas. Desde finales de los años 80 del siglo XX, las bibliotecas españolas han vivido un proceso de modernización y de compromiso con sus públicos. Educación y biblioteca alentó esos cambios y aglutinó una corriente de biblioteconomía comprometida con la acción social de las bibliotecas, los valores del servicio público y la democratización de la cultura. Durante sus años de existencia, Educación y biblioteca estuvo abierta a todos los profesionales bibliotecarios que se sirvieron de ella como de un foro en el que discutir proyectos y poner en común buenas prácticas. Siendo un proyecto privado e independiente ha supuesto una experiencia de trabajo colaborativo y de funcionamiento en red.

\section{Palabras clave}

Bibliotecas públicas, Acción social de las bibliotecas, Trabajo colaborativo, Revistas.

\section{Title: Educación y biblioteca, a unique professional journal}

\begin{abstract}
The journal Educación y biblioteca (Education and the library) accompanied the Spanish public libraries in the great change that took place over three decades. Since the late 80 s of the 20th century, Spanish libraries have undergone a process of modernization and engagement with their audiences. Educación y biblioteca encouraged these changes and brought together a stream of librarianship committed to social action by libraries, public service values and the democratization of culture. During its existence, Educación y biblioteca was open to all librarians, who used it as a forum to discuss projects and share best practices. As a private and independent project it offered a collaborative work experience and networking.
\end{abstract}

\section{Keywords}

Public libraries, Social activity of libraries, Collaborative work, Journals.

Pérez-Iglesias, Javier (2013). "Educación y biblioteca, una revista profesional particular". El profesional de la información, marzo-abril, v. 22, n. 2, pp. 139-142.

\section{Introducción}

Mis años en la Dirección de Educación y biblioteca, desde septiembre de 1996 hasta diciembre de 2000, fueron de una gran actividad. Al mirar hacia atrás recordando aquel tiempo, veo una sucesión acelerada de lecturas, viajes, emails, discusiones, cursos, jornadas, entrevistas, reuniones, fotomecánicas, imprentas, mesas redondas, colegios, facultades, bibliotecas de barrio, de pueblo, bibliobuses, horas y horas frente al ordenador y, por una extraña elasticidad del tiempo pasado, una vida social intensa. Conservo pocas fotos pero en las que hay de esos años se me puede ver con distintos colores de pelo, diferentes gafas y muy diversos atuendos. Todo estaba en permanente cambio. También cambiaban las bibliotecas públicas que parecían entrar en un período interesante. Se creaban espacios, se implantaban nuevos servicios, se buscaban aliados, todo con un afán de modernizar la vida cultural del país. 
Creo sinceramente que lo que voy a contar aquí se puede hacer extensivo a los años previos a mi trabajo en la revista $y$, desde luego, a los que siguieron después de que yo dejara la Dirección. Lo que es irme no llegué a irme nunca porque seguí colaborando con el equipo, publicando reseñas y artículos y, sobre todo, en permanente contacto con toda esa red de personas que se había ido tejiendo en torno a Educación y biblioteca. EyB como la llamábamos los íntimos para abreviar, y me voy a tomar la licencia de referirme así a ella en este texto.

Una revista para bibliotecarios, con contenido cultural, línea independiente, y una clara tendencia por los temas incómodos

Es difícil creer que EyB se hiciera con tan pocas personas en plantilla. En aquel tiempo se publicaba cada mes, con una media de 200 páginas, y aparecía llena de experiencias de bibliotecas de todo el estado español, de reseñas de recursos bibliográficos y de novedades técnicas, de reflexiones y artículos de pensamiento. En cada número se presentaba un panorama crítico de la oferta editorial infantil y juvenil, con cuidado de que no se redujera a novelas y cuentos, con mucho espacio para el libro de conocimiento, los álbumes (entonces sin tanto hueco en el mercado), la poesía, el teatro y la tradición oral. Además, se dedicaba una parte importante de las páginas a un dossier sobre un tema de interés para las bibliotecas públicas y escolares. Todo eso con un equipo de dos personas en la Redacción, una administrativa y otra persona dedicada a la búsqueda de publicidad.

Trabajábamos en el trapecio que suponía hacer una revista para bibliotecarios, con contenido cultural, con una línea independiente, con una clara tendencia por los temas incómodos (drogas, orientaciones sexuales diversas, multiculturalidad...) y los acercamientos excéntricos (anti-prohibicionismo, respeto por la diferencia, pedagogías críticas, investigación-acción...) sin casarnos con nadie (no era extraño que alguna editorial de las grandes marcara nuestro número de teléfono para protestar por una reseña "inconveniente"). La línea editorial nos situaba en lo que se ha dado en llamar biblioteconomía progresista pero tanto nuestros seguidores/lectores como muchos de los contenidos no eran fácilmente clasificables.

\section{Un proyecto colaborativo}

No hubiera sido posible realizar $E y B$, y que se mantuviera en el tiempo, sin toda la red de apoyo que se distribuía por toda España y alcanzaba a otros países al otro lado del Atlántico. Era una red social y profesional anterior a las redes sociales, tal como se definen desde la web 2.0, pero con algunas similitudes. No siempre nos conocíamos personalmente cuando empezábamos a interactuar. Había gente que se ponía en contacto con la Redacción para hacernos una propuesta y, otras veces, era desde EyB de donde partía la solicitud. El caso es que se crearon lazos profesionales, bastante duraderos, antes de que pudiéramos coincidir en vivo. Algunas de esas relaciones derivaron hacia amistades personales y todavía disfrutamos de ellas. En fin, nada que no ocurriera desde que la gente puede escribirse cartas y existe un servicio de reparto fiable, aunque en nuestro caso el medio se llamara correo electrónico. Pero lo que quiero resaltar aquí es el modelo de trabajo colaborativo (cada número se construía desde más de un lugar físico y con la participación de personas de distintos ámbitos e instituciones) y la amplitud de la visión. Teníamos la sensación de que creábamos opinión, pero no quienes trabajábamos en la sede de la revista, sino todos los que la construíamos desde tantos lugares diferentes.

Siendo como éramos un proyecto tan distribuido y tan "líquido" no me explico cómo no nos convertimos en una publicación electrónica, en una mezcla de newsletter y blog, accesible en la web. Bueno, ese "no me explico" es retórico, me lo explico perfectamente. No teníamos dinero, ni un duro para poder reinvertir en la revista. Cuando llegué a $E y B$ el cd-rom seguía siendo una tecnología interesante y queríamos digitalizar los números atrasados (algunos ya agotados) para ofrecerlos a un precio módico a suscriptores y admiradores. No hubo manera. Se tardaron años en conseguir la financiación para digitalizar la revista. El equilibrio del trapecio es lo que tiene.

EyB era un proyecto privado pero pobre. Un experimento, una apuesta personal sin apoyo institucional estable. Es verdad que se necesitaban las suscripciones que mantenía el Ministerio de Cultura y las compras que se realizaban desde las consejerías de las comunidades autónomas para sobrevivir. Teníamos una cierta dependencia de las administraciones que, seguramente, nos hizo vivir por encima de la miseria para acabar muriendo, como cabecera, por encima de nuestras posibilidades.

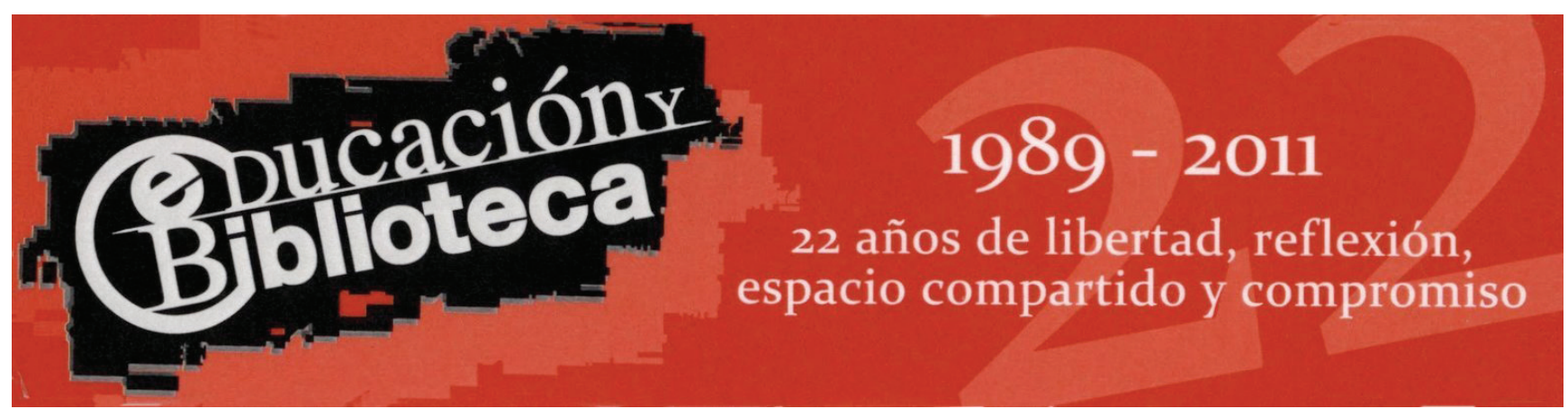

Marcapáginas Educación y biblioteca 
El caso es que $E y B$ se convirtió en una red, un entramado de relaciones, que hacía posible que cada mes saliera de la imprenta y llegara a manos de los suscriptores (personas e instituciones) y participara en la vida profesional creando tendencia entre la comunidad bibliotecaria española. Desde sus páginas se presentaba y divulgaba una idea de biblioteca comprometida con su comunidad, pendiente de las necesidades de sus usuarios, atenta a quiénes solicitaban sus servicios y a los que, por un motivo u otro, no se acercaban nunca. Una biblioteca que se adelanta, que mira hacia fuera y sale fuera. Muchos servicios que ahora nos resultan habituales dieron sus primeros pasos en numerosos centros alentados por la revista: bebetecas, información a la comunidad, ofertas de formación en competencias de información, utilización de los espacios y colecciones al servicio de los grandes retos de la sociedad (sida, inmigración, igualdad de género, drogas, libertad de opción sexual, etc.). Por la manera de abrirse a la comunidad bibliotecaria, y de ofrecer sus páginas como un lugar de encuentro y de intercambio de ideas, EyB acabó creando una comunidad y una manera de entender las bibliotecas. El asociacionismo, la deontología profesional, la censura, el compromiso social, los derechos de autor, eran temas que saltaban de las bibliotecas a la revista con un ida y vuelta.

Como aventura editorial era un caso curioso porque no había otro ejemplo de revista profesional independiente de cualquier asociación o administración. Es verdad que había una Asociación Cultural Educación y Bibliotecas detrás pero, después de unos pocos años, era la publicación la que tiraba del proyecto y aglutinaba voluntades.

\section{Indefinidos y comprometidos}

Sin ser una revista científica, no tuvo nunca esa pretensión, sí que se publicaron contenidos que hubieran sido admitidos como artículos científicos en cualquier otra publicación. Entre otras cosas, hubo siempre una fuerte conexión con los nacientes estudios de Biblioteconomía y Documentación y con la comunidad investigadora que se estaba formando en torno a esa disciplina. Así, cuando ya llevaba varios años en circulación y era vista desde algunos sectores como un reducto para "bibliotecarios perroflautas" avant la lettre, se construyeron dosieres sobre marketing, digitalización, últimas tendencias en catalogación, recursos en internet, etc.

\section{Ey $B$ se convirtió en una red, un entrama-} do de relaciones

Mención especial merecen todas las invitaciones a gente que, sin ser de la profesión, podía aportar una visión interesante sobre el quehacer bibliotecario. Por las páginas de $E y B$ han desfilado dejando sus ideas y su visión sobre las bibliotecas personas que se dedicaban a la música, edición, ciencias de la salud, filosofía, matemáticas, escritura, artes plásticas, radio, gestión cultural o a los clubes de lectura, por poner algunos ejemplos.

Como el trapecio no nos dejaba estar quietos, dedicamos mucho tiempo al diseño de las portadas, que discutíamos

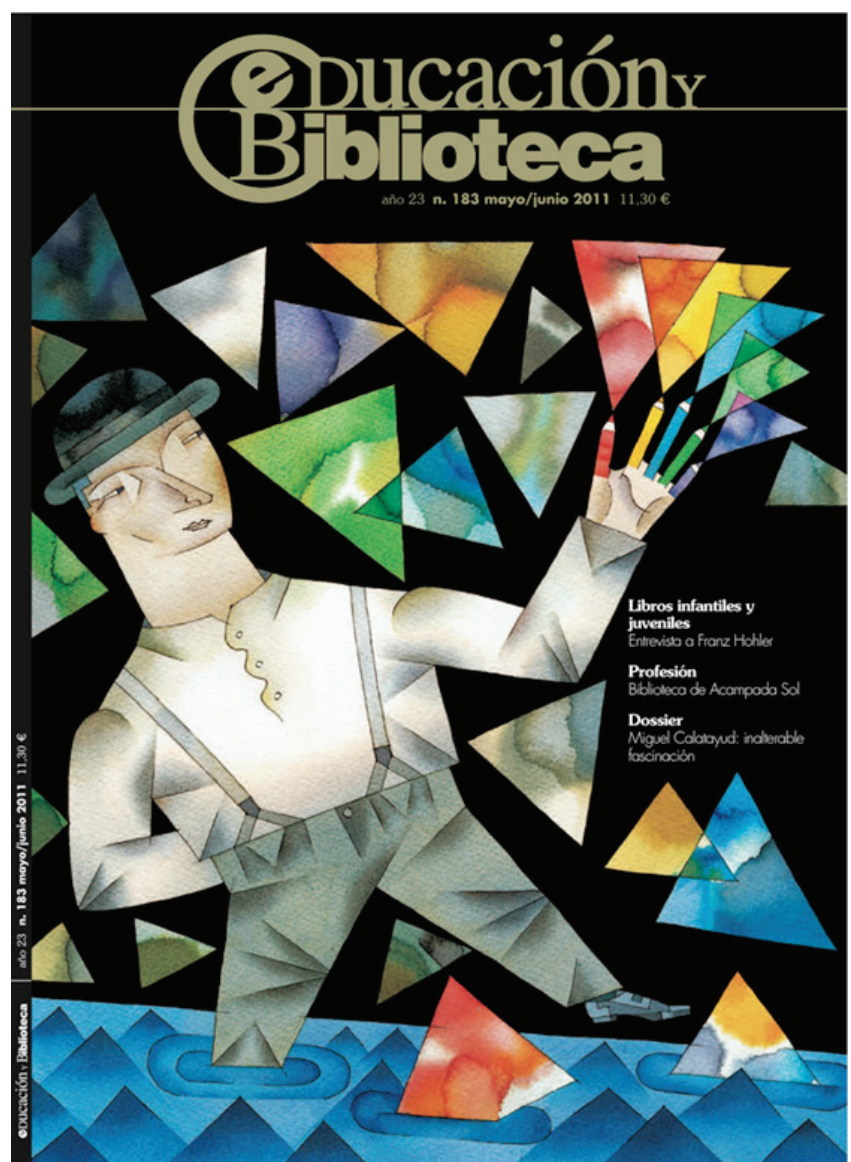

Último número de Educación y biblioteca, mayo-junio de 2011

con el artista encargado de hacerlas. Normalmente queríamos una interpretación visual del contenido del dossier, así que le pasábamos algunos textos al diseñador y luego teníamos una primera reunión aproximativa. A veces eran necesarias más reuniones y aprovechábamos para hacer un diseño especial de alguna de las páginas de la revista. En los últimos años de vida de EyB se pudo contar con la colaboración de importantes figuras del mundo del libro ilustrado.

EyB nació a finales de la década de los 80 , con un panorama bibliotecario bastante triste, y ha desparecido en un momento en el que las bibliotecas corren un serio en peligro. Las bibliotecas que se empeñaba en inventar EyB eran lugares en los que cada persona era tratada como un huésped de lujo, como alguien especial que merecía los mayores cuidados y las más delicadas atenciones. Hoy, esas bibliotecas se han convertido en algo indeseable para las administraciones porque la mayoría de la población somos considerados como chusma y el objetivo es no gastar en nosotros.

Los bibliotecarios españoles hemos tenido que mirar fuera o lejos para buscar referentes. Durante años ha sido fuera de nuestras fronteras en donde visitábamos edificios y modelos de organización que nos sirvieran de inspiración. Íbamos a Gran Bretaña, a los países nórdicos, a los Estados Unidos, a Francia..., para alimentar nuestras ansias de bibliotecas útiles, al servicio de las necesidades de las personas. Más tarde pudimos buscar en el pasado, en la corta primavera republicana, para encontrar modelos de profesión estimulantes. Quizá ahora, con este regreso a la pérdida de derechos y a los presupuestos magros, debamos buscar en otros 




Algunos asistentes al homenaje a la revista Educación y biblioteca (Facultad de Ciencias de la Documentación de la Universidad Complutense de Madrid, 16 nov. 2011): José-Antonio Merlo, Inmaculada Vellosillo, Javier Pérez-Iglesias, Blanca Calvo, Marta Martínez-Valencia, María-Jesús Del-Olmo, Ana Santos, Pedro López-López y José-Antonio Gómez-Hernández. http://www.elprofesionaldelainformacion.com/notas/homenaje-a-la-desaparecida-revistaeducacion-y-biblioteca

lugares donde llevan muchas décadas conviviendo con esa situación que llamamos crisis, para encontrar respuestas y ánimos. Se me ocurre que puede ser necesaria una cooperación Sur-Norte que nos ayude a ver otros caminos.

Presentaba y divulgaba una idea de biblioteca comprometida con su comunidad

\section{Y llegó la despedida}

Lo cierto es que las bibliotecas han sabido adaptarse a muchas situaciones y, la verdad, trabajar con presupuestos exiguos ha sido la norma en tiempos no tan lejanos. Hay muchos ejemplos bibliotecarios de aprovechar recursos y de hacer más con menos (aunque todo tiene un límite) así que eso nos permite ser medianamente optimistas para pensar que no todo van a ser maquinarias oxidadas. Quizá haya que inventar otra $E y B$ para contar todo esto.

Durante los años de su existencia $E y B$ fue un reflejo de la realidad bibliotecaria. De lo que podíamos, de lo que soñá- bamos y de lo que queríamos que pudiera ser. Para mí fue un honor poder llegar a dirigir esa revista que leía y admiraba. Luego, ha sido un orgullo continuar unido a ella en los años siguientes a mi etapa como director. ¡Qué gusto haber podido asistir al crecimiento y desarrollo de un proyecto en el que uno pudo poner un eslabón! ¡Qué satisfacción recibir en casa cada nuevo número y que me sorprendiera y estimulara su contenido!

Me despido con algunos nombres que me han venido a la cabeza mientras escribía este texto. Seguro que me dejo a mucha gente fuera pero éstos son los que me han visitado y me ayudan a despedirme con un hasta luego: Antonia Ontoria, Ramón Salaberria, Ana Garralón, Luisa Mora, Paco Solano, Cristina Ameijeiras, Blanca Calvo, Inés Miret, Carmen Gómez-Varela, Margarita Pérez-Pulido, José-Antonio Merlo, José-Antonio Gómez-Hernández, Michéle Petit, Gustavo Puerta-Leisse, José-Manuel Nuevo, Gelo Quero, Sara Huete, Nona Domínguez-Sanjurjo, Mercè Escardó, Ana Párraga, Ana Castillo, Lourdes Rodríguez, Juana Abellán, Samuel AlonsoOmeñaca, Raquel López-Royo, Villar Arellano, María-Jesús Del-Olmo, Esther Martínez-Olmo, Eva Méndez, Pedro López, María-Jesús Rodríguez, Inmaculada Vellosillo, Luis-Miguel Cencerrado, Félix Benito-Morales, Daniel Goldin, Sol Ugarte, Marta Martínez-Valencia...

\section{Suscripción EPI sólo online}

Pensando sobre todo en los posibles suscriptores latinoamericanos, ya no es obligatorio pagar la suscripción impresa de EPI para acceder a la online.

EPI se ofrece a instituciones en suscripción "sólo online" a un precio considerablemente más reducido (96,69 +21\% IVA euros/año), puesto que en esta modalidad no hay que cubrir los gastos de imprenta ni de correo postal. 